\title{
DECOUPLING IN THEORIES WITH ANOMALIES
}

\author{
T. STERLING \\ University of Michigan, Department of Physics, Ann Arbor, MI 48109, USA \\ M. VELTMAN \\ University of Michigan, USA \\ and \\ Inst. for Theor. Physics, Rijksuniversiteit, Utrecht, The Netherlands
}

Received 9 March 1981

\begin{abstract}
We consider the appearance of infinities due to the triangle anomaly in the Weinberg model by introducing terms in the lagrangian which restore the Ward identities. It is then shown that these are not the same as the divergences which occur in the standard model if the quarks (which cancel the anomalies) are given a large mass. We have not succeeded in finding a model in which the infinities due to the anomalies are the leading effects of heavy fermions.
\end{abstract}

\section{Introduction}

The decoupling theorem [1] is of great importance in establishing which theories are viable as ultraviolet theories given our present day knowledge of the infrared (infrared is everything below $300 \mathrm{GeV}$ ). In its original form this theorem states that the influence of heavy particles on the infrared theory is unobservable, i.e. it can be absorbed in the parameters of the infrared theory, if both the infrared and ultraviolet theories are renormalizable. For instance, weak interactions mediated by vector bosons with a mass of about $100 \mathrm{GeV}$ can be ignored in QED calculations concerning the Lamb shift and anomalous magnetic moments. As such, the decoupling theorem has been taken for granted by two generations of physicists.

But in the context of gauge theories decoupling is by no means evident. Furthermore, the concept of naturalness reveals a domain of interest beyond the scope of the original formulation. For instance, one may inquire whether the influence of the heavy particles on the parameters of the infrared theory is unnatural, that is requires fine tuning. Thus, in a grand unified theory such as $\mathrm{SU}(5)$, the radiative corrections to the low lying masses could be much larger than the observed mass which would be unnatural. Actually, the recent work of Kazama, Unger and Yao [2] has shown that this is not the case, thereby extending the notion of decoupling. Other cases of interest are the influence of heavy fermions on the $\rho$ parameter of the standard 
model and the possibility that the Higgs scalar is very heavy. In all these cases one goes beyond the scope of the decoupling theorem and tries to establish which effects of the heavy particles remain in the infrared. The interest in such sensitivity to very heavy masses is, of course, evident.

In this article we consider another case not covered by the decoupling theorem. Imagine the standard model with only one generation of fermions, containing the electron and its neutrino, and the up and down quarks. If we remove the quarks from that theory then we are left with Weinberg's original model of leptons, which is in fact non-renormalizable because it contains anomalies. Thus, the decoupling theorem is not applicable and if we increase the mass of the quarks to large values then we expect to see observable effects in the lepton sector. The question is: how strong are these effects? Naively one would expect the following. Consider the Weinberg model [3] with its anomalies. The first non-renormalizable infinity occurs at the two-loop level and it appears as a logarithmic divergence. Consequently, one would think that in the model including quarks the quark mass would show up in the lepton sector only at the two-loop level.

In actual fact this is not the case. Amazingly, it seems to be impossible to construct a model that shows decoupling at the one-loop level, and in this article we describe some efforts in this direction.

The consequence of this situation is that indeed anomalies must be absent in the infrared theory, even if the ultraviolet theory is not very far away. In a tumbling scheme [4], anomalies should be absent at every stage, and 't Hooft's [5] work on the constraints following from the requirement of absence of anomalies is relevant at all stages.

In sect. 2 we consider the pure Weinberg model and establish the whereabouts of the non-renormalizable infinities. In sect. 3 we consider the one generation standard model, and investigate what happens if the quark masses are made large. In sects. 4 and 5 we show how two possible ways of evading the difficulties do, in fact, not work.

\section{Infinities of the Weinberg model}

Consider the Weinberg model with only an electron and a neutrino in the so-called unitary gauge. If there were no anomalies all nonrenormalizable infinities would cancel in the $S$-matrix. But due to the anomaly at the one-loop level the symmetry will be broken and the mechanism of cancelling divergences is disturbed. Consequently, at some point, at the earliest at the two-loop level, certain nonrenormalizable divergences will remain. The question is "where?"

If we tried to pursue a calculation in the way described above, that is starting from the unitary gauge, then the analysis would become quite difficult and opaque. Instead we will proceed as follows. We start from the theory in the Feynman't Hooft gauge where renormalizability seems good. However, due to the anomalv 
the Ward identities break down and unitarity is violated. We then introduce new terms in the lagrangian such that the Ward identities, and thus unitarity, are restored. But these new terms are of a non-renormalizable type and they give rise to infinities that can be found rather easily.

To do the calculation we must specify the regularization scheme to be used, in particular, we must specify how to treat $\gamma^{5}$. We will use the scheme of ref. [6] in which $\gamma^{5}$ is taken to be the product of the first four $\gamma$ matrices. Furthermore, the Feynman rules are as specified in ref. [7]. In the gauge specified, for example, one relevant Ward identity concerns the amplitude for a neutral vector boson to decay into two photons, and multiplying this amplitude with $k_{\mu}$, the $\mathrm{W}^{0}$ four-momentum, the result must be equal to $i M_{0}$ times the amplitude for a $\phi^{0}$ (the neutral Higgs ghost) decay into two photons. Due to the anomaly this is not the case but we can restore this and other Ward identities by introducing a term [8] into the lagrangian:

$$
\frac{i g^{3} \tan \theta_{\mathrm{W}}}{128 \pi^{2} M} \varepsilon_{\mu \nu \alpha \beta}\left(F_{\mu \nu} G_{\alpha \beta}^{a} \phi^{a}+F_{\mu \nu} F_{\alpha \beta} \phi^{0} \tan \theta_{\mathrm{W}}\right)
$$

(this term is derived in appendix A), where $F_{\mu \nu}$ is the field strength of the original $U(1)$ vector boson, and $G_{\mu \nu}^{a}$ is the field strength of the $\operatorname{SU}(2)$ vector bosons and $\phi^{a}$ is the unphysical Higgs. $\theta_{\mathrm{W}}$ is the weak mixing angle. Note that this term is of order $g^{3}$.

We must now investigate the divergences arising from the term (2.1). This may happen if this term is part of a loop, either of the self-energy or triangle type. Using vertex (2.1) only once there are several logarithmically divergent amplitudes, of a non-renormalizable type. One finds an anomalous magnetic moment for the electron (fig. 1a) and the W (fig. lb) and similar moments for other vector-lepton and vector-vector-vector interactions. Infinities are also found in lepton-lepton and lepton-vector scattering, for example figs. 2a,b. Since the vertices (2.1) are in fact implicit one-loop graphs, these diagrams must be seen as two-loop diagrams. Thus logarithmic divergences appear at the two-loop level. At the three-loop level quadratic divergences (poles for $n=2$ ) appear, such as in the well-known diagram of fig. 3 .

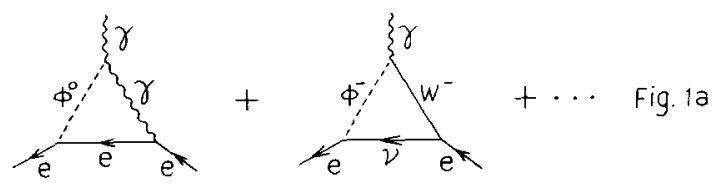

and the $w$

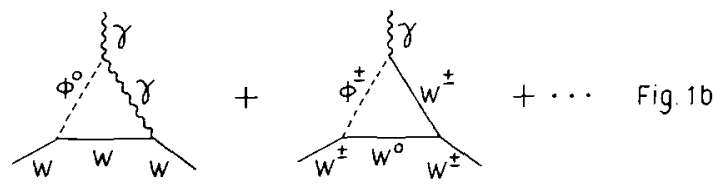

Fig. 1. 


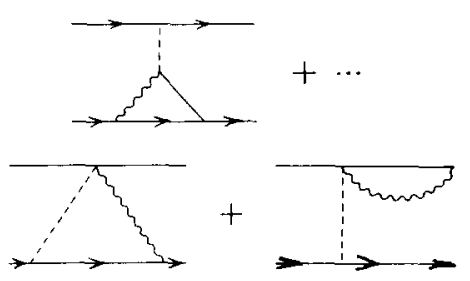

Fig. 2 .

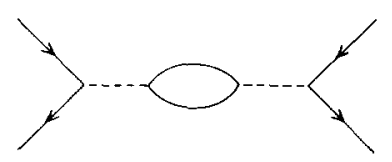

Fig. 3 .

\section{The Weinberg model with quarks}

We have shown that the triangle anomaly causes non-renormalizable divergences in the two-loop approximation. We would like to relate these divergences to a physical parameter which mimics them as it gets large. A model ready made for this is the standard [9] model of quarks and leptons. If the quark mass was made large and the quarks decoupled (as they do at the tree level), the remaining theory would be the Weinberg model. Since the Weinberg model is not renormalizable this decoupling cannot occur. We want a theory in which the non-decoupling is evident only through the anomaly, that is in the two loop effects of the previous section. However, even in the one-loop approximation there are effects which grow with increasing quark mass.

Hung and Wolfram and Politzer [10] have discussed the effect of heavy fermions on the effective Higgs potential. In the standard model the potential is

$$
V_{\text {eff, fermions }}=-4 g^{4} \sum_{\text {fermions }}\left(\frac{m_{\mathrm{f}}}{M}\right)^{4} \phi^{4} \ln \phi^{2} .
$$

Clearly, if this term is larger than other $\phi^{4} \ln \phi^{2}$ terms, the potential is unbounded below and the vacuum is unstable at any local minimum. This makes any attempt to let the fermion mass be large suspicious but the potential may have significant contributions from higher loops that could give a minimum in the potential. For example, if the two-loop contribution has a term

$$
\phi^{4}\left(\ln \phi^{2}\right)^{2}
$$

with a positive coefficient, this would overcome the $\phi^{4} \ln \phi^{2}$ term at sufficiently high $\phi^{2}$. 


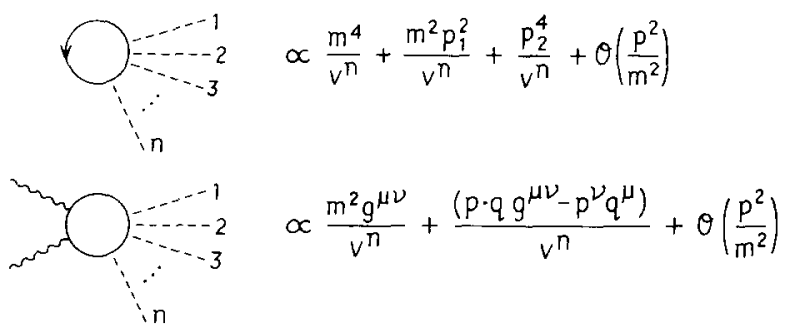

Fig. 4.

Assuming that the potential somehow has a minimum we can then find where the quark mass enters other one-loop corrections. The source of all the problems are graphs with all external lines being Higgs bosons or graphs with two external vector bosons and one Higgs, shown in fig. 4 ( $m=$ heavy fermion mass, $V=$ vacuum expectation value), as previously discussed by Chanowitz, Furman and Hinchliffe [11]. We show in fig. 4 the type of terms encountered in these diagrams expanded as a power series in $p^{2} / m^{2} . p_{1}$ and $p_{2}$ represent more complicated functions of the external momenta. Some diagrams with small $n$ are calculated in appendix $C$.

Some of these graphs (those with less than 4 external lines) can have infinite pieces that must be renormalized. There are also finite pieces which do not decrease as the mass increases; it is these terms which exhibit most clearly the lack of decoupling at one loop. Appendix $\mathrm{C}$ shows the calculation of some of these graphs. Of course, most of these terms which diverge with increasing $m$ have nothing to do with the anomalies. We have attempted to construct a theory which has the Weinberg model as a low energy limit and has only the anomalous divergences as low energy manifestations of some heavy particles and we have failed.

\section{Extra Higgs bosons}

We have seen that letting the $\mathrm{u}$ and $\mathrm{d}$ masses become large in the standard model creates large effects at the one loop level. We would like to find a model in which the infinities due to the anomalies (sect. 2) are the leading effects of the ultraviolet part of the theory. In order to do this we must somehow cancel all the effects shown in sect. 3. There are two different problems. The first is the vacuum instability caused by fermion loops in the effective Higgs potential. This problem can be solved by adding some scalars to the theory which are at least as heavy as the fermions; since the scalar loops have the opposite sign in the effective potential we can restore the potential to its original form. The second problem is that there are effects (at low energy, that is less than $300 \mathrm{GeV}$ ) which do not decrease as the heavy fermion mass increases. In the one-loop approximation these effects involve external Higgs bosons (the Higgs boson must be part of the infrared theory). A more complicated Higgs sector is able to avoid some of these problems, but introduces new problems. 
In order to evade the vacuum instability we can add more scalars. In fact, by giving the added scalars the same $\mathrm{SU}(2) \times \mathrm{U}(1)$ structure as the heavy fermions we can eliminate all effects on the effective potential in the one-loop approximation. A way to see this is to consider the lagrangian

$$
\begin{aligned}
\varrho= & +A^{+} \partial^{2} A+F^{+} F-\bar{\psi}_{\mathrm{L}} \partial \psi_{\mathrm{L}}+a_{j}^{+} \partial^{2} a_{j}+f_{j}^{+} f_{j}-\bar{\psi}_{\mathrm{R} j} \partial \psi_{\mathrm{R} j} \\
& -G\left[\left(\bar{\psi}_{\mathrm{L}} H\right) \psi_{\mathrm{R} 1}+\left(A^{+} H\right) f_{1}+\left(F^{+} H\right) a_{1}\right]+\text { h.c. } \\
& -G\left[\left(\bar{\psi}_{\mathrm{L}} \tilde{H}\right) \psi_{\mathrm{R} 2}+\left(A^{\tilde{f}} H\right) f_{2}+\left(F^{\tilde{+}} H\right) a_{2}\right]+\text { h.c. },
\end{aligned}
$$

where $j=1,2 ; A, \psi_{\mathrm{L}}$ and $F$ are $\mathrm{SU}(2)$ doublets and $a, \psi_{\mathrm{R}}$ and $f$ are singlets. $H$ is an $\mathrm{SU}(2)$ constant, $\left(A^{+} H\right)$ and $\left(A^{+} H\right)$ are the two SU(2) products of $A^{+}$and $H$. This lagrangian obeys a supersymmetry:

$$
\begin{aligned}
& \delta A=\bar{\alpha}_{\mathrm{R}} \psi_{\mathrm{L}}, \quad \delta \psi_{\mathrm{L}}=(\partial A) \alpha_{\mathrm{R}}+F \alpha_{\mathrm{L}}, \quad \delta F=\bar{\alpha}_{\mathrm{L}} \partial \psi_{\mathrm{L}} ; \\
& \delta a_{j}=-\bar{\alpha}_{\mathrm{L}} \psi_{\mathrm{R} j}, \quad \delta \psi_{\mathrm{R} j}=-\left(\partial a_{j}\right) \alpha_{\mathrm{L}}-f_{j} \alpha_{\mathrm{R}}, \quad \delta f_{j}=-{ }^{-} \alpha_{\mathrm{R}} \partial \psi_{\mathrm{R} j},
\end{aligned}
$$

where $\alpha$ is an infinitesimal spinor. The $F$ and $f_{j}$ fields can be eliminated to give a lagrangian with only dynamical fields. Notice that the supersymmetry is good only if $H$ is a constant because the supersymmetry depends on dropping a total derivative from the lagrangian. However, even if we introduce a kinetic term for $H$, supersymmetric cancellations will occur for diagrams with $H$ constant, that is with zero momentum. Specifically, the one-loop effective potential has no contribution from the supersymmetric fermion-boson pair. The breaking of the supersymmetry makes it unnatural that the fermion and boson fields couple to $H$ with the same strength, $G$, but if we allow this unnatural condition to be satisfied the effect of the fermions disappears in the one-loop effective potential.

In the two-loop approximation this cancellation will not occur because there are graphs with internal $H$ lines. In the limit that the mass of the heavy fermions, $m$, is large these graphs will dominate because they have the most factors of $m^{2}$ from the Higgs coupling. Using the method of Lee and Sciaccaluga [12] we can calculate the contribution to the potential from the diagrams of fig. 5:

$$
V=\frac{2(g m)^{6}}{(8 \pi)^{4} M^{6}} \phi^{4}\left(\left(\ln \phi^{2}\right)^{2}+A \ln \phi^{2}\right),
$$

where $A$ depends on the renormalization point. Since $V$ gets large with $\phi^{2}$ this will not cause vacuum instability.

This eliminates the vacuum problem but the fermion boson pair will not decouple. There are several places where this can be observed. First, the Higgs propagator has 


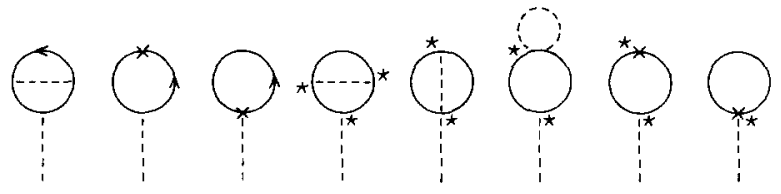

Fig. 5 .

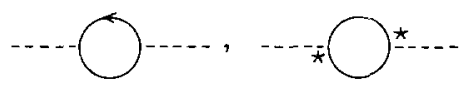

Fig. 6.

finite corrections due to the graphs of fig. 6 . These graphs can be expanded as a power series in $p^{2} / m^{2}$ :

$$
\Gamma=A+B p^{2} / m^{2}+C p^{4} / m^{4}+\cdots .
$$

$A$ and $B$ are infinite and will be absorbed in the renormalization but $C$ is $\mathrm{O}\left(\mathrm{m}^{4}\right)$ and so the $p^{4}$ term is independent of mass. As shown in appendix $C$, the fermion and boson contributions to $C$ have the same sign so there is no way to arrange them to cancel. Similarly the decay of the Higgs boson into two photons through a fermion and boson loop (fig. 7) is independent of the mass [13] and the scalar loop contributes with the same sign as the fermion.

These, and other problems due to the large coupling of the Higgs to the heavy fermions (and bosons) could conceivably be remedied by adding a second Higgs doublet which would couple only to the heavy fermions while the other couples only to the light fermions, each set having its own global $\mathrm{SU}(2)$ to prevent other possible couplings. At the tree level we already see effects on the infrared theory. Specifically the mass of the charged $\mathrm{W}$ bosons will be

$$
M^{2}=g^{2}\left(V_{1}^{2}+V_{2}^{2}\right) .
$$

The extra Higgs must be in the ultraviolet part of the theory so its contribution to the $\mathrm{W}$ mass is unaccounted for in the infrared theory. This extra Higgs would also result in pseudo-Goldstone bosons which are massless at the tree level; these would only get a mass proportional to $m$ at the two-loop level.

The basic problem is that in order to contribute to the anomaly the fermions must have a non-trivial SU(2) structure and therefore any Higgs which gives them mass

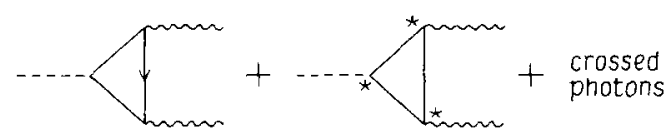

Fig. 7 
must also give the $\mathrm{W}$ bosons mass and thereby influence the low energy theory. So the only Higgs we can have is the one already in the theory and we have already seen the effects a heavy fermion has on its interactions.

\section{Fermion mass mixing}

The Higgs mechanism cannot give the $\mathrm{u}$ and $\mathrm{d}$ a large mass in a satisfactory way. Therefore, we must try to find a different way to make them massive. In $\mathrm{SU}(2) \times \mathrm{U}(1)$ models there can be massive fermions without spontaneous symmetry breaking. These fermions must either be in a pair, namely a left- and a right-handed fermion which are in a single representation of $S U(2)$ and have the same $U(1)$ charge, or in SU(2) singlets with no $U(1)$ charge; in the second case there can be a Majorana mass. In either case there is no anomaly. The possibility arises that such particles could mix with other fermions which have an anomaly in such a way that both acquire a mass. However, in the case where these anomaly free masses get large the mixing due to relatively small vacuum expectation values is small and the anomaly free particles will decouple.

A familiar example of this type of fermion is the addition of a right handed "neutrino" to the Weinberg model, that is an SU(2) singlet with no U(1) charge. This particle can both have a Majorana mass and mix with the electron neutrino as a result of the Higgs vev. The Higgs fermion sector of the lagrangian is

$$
\begin{array}{r}
\frac{m_{\mathrm{c}}}{V}(\bar{e} \bar{\nu})_{\mathrm{L}}\left(\begin{array}{c}
\phi_{0} \\
\phi^{+}
\end{array}\right) e_{\mathrm{R}}+\text { h.c. }+\frac{m_{\nu}}{V}(\bar{e} \bar{\nu})_{\mathrm{L}}\left(\begin{array}{c}
-\phi^{-} \\
\phi_{0}
\end{array}\right) \nu_{\mathrm{R}}+\text { h.c. }+M \nu_{R}^{c} v_{R}, \\
\langle\phi\rangle=V .
\end{array}
$$

The mass terms are (note that $\nu_{\mathrm{R}}^{\mathrm{c}}$ is left handed),

$$
\left(e_{\mathrm{L}} \nu_{\mathrm{L}} \nu_{\mathrm{R}}^{\mathrm{c}}\right)\left(\begin{array}{ccc}
m_{\mathrm{e}} & 0 & 0 \\
0 & 0 & \frac{1}{2} m_{\nu} \\
0 & \frac{1}{2} m_{\nu} & M
\end{array}\right)\left(\begin{array}{c}
e_{\mathrm{R}} \\
\nu_{\mathrm{L}}^{\mathrm{c}} \\
\nu_{\mathrm{R}}
\end{array}\right)+\text { h.c. }
$$

When $m_{v} \ll M$ the mass eigenstates are approximately

$$
\begin{aligned}
& \phi_{1}=\nu_{\mathrm{L}}+\nu_{\mathrm{L}}^{\mathrm{c}}-\frac{m_{\nu}}{2 M}\left(\nu_{\mathrm{R}}+\nu_{\mathrm{R}}^{\mathrm{c}}\right), \quad \text { with mass } m_{v}^{2} / 4 M, \\
& \phi_{2}=\nu_{\mathrm{R}}+\nu_{\mathrm{R}}^{\mathrm{c}}+\frac{m_{\nu}}{2 M}\left(\nu_{\mathrm{L}}+\nu_{\mathrm{L}}^{\mathrm{c}}\right), \quad \text { with mass } M .
\end{aligned}
$$

We see that as $M$ gets large the left-handed neutrino mass gets very small and the right-handed component will be very massive. In this situation the decoupling 
theorem will hold and there will be no noticeable effect of the $\nu_{\mathrm{R}}$ at energies much less than its mass.

In more complicated mass matrices the result is essentially the same; the particles which can be massive decouple if their masses are large. Therefore, there is no possibility of making massive fermions which have an anomaly by such a method.

\section{Conclusion}

In this paper we have shown that fermions that have a mass acquired by a vacuum expectation value which breaks $\mathrm{SU}(2) \times \mathrm{U}(1)$ symmetry will not decouple as their mass gets large. Furthermore, this non-decoupling is not due to the anomalies which guarantee that fermions with anomalies cannot decouple. It seems that it is impossible to construct a model in which the anomalies provide the leading evidence of non-decoupling. The anomalies cause divergences only in two-loop diagrams whereas heavy fermions will have one-loop diagrams which diverge with the fermion mass.

Since the largest effects which are seen as the fermion masses get large are not related to the anomaly there are wider consequences than forcing the anomalies to be cancelled by reasonably light fermions. There can be no fermions with masses much heavier than $300 \mathrm{GeV}$ whose mass breaks $S U(2) \times U(1)$ without leaving observable effects at lower energies. These effects would appear in different ways depending on the Higgs mass and the complexity of the Higgs sector of the theory.

We are indebted to William Bardeen, David Unger and Y.-P. Yao for instructive comments.

\section{Appendix A}

\section{TRIANGLE DIAGRAM}

As an example there are two anomalous diagrams, shown in fig. 8. The second diagram obtains by a photon interchange from the first. The contribution of the first diagram is:

$$
\begin{aligned}
\Gamma_{I}^{\alpha \mu \nu}= & \frac{i g^{3} s_{\theta}^{2}}{4 C_{\theta}} \\
& \times \int \mathrm{d}_{n} r \frac{\operatorname{Tr}\left[\gamma^{5} \gamma^{\alpha}\{-i \gamma(p+r)+m\} \gamma^{\mu}(-i \gamma r+m) \gamma^{\nu}(-i \gamma(r-q)+m\}\right]}{\left.\left(r^{2}+m^{2}\right)\left((r+p)^{2}+m^{2}\right)(r+p+k)^{2}+m^{2}\right)} .
\end{aligned}
$$

The notation is as in ref. [7]. To work out the trace it is expedient to move factors 


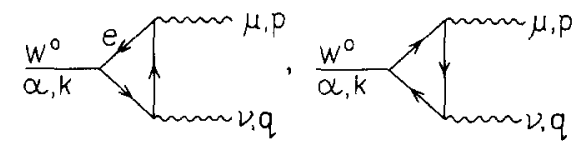

Fig. 8.

$-i \gamma r+m$ to the left or right, such as to obtain the combination $(-i \gamma r+m)(i \gamma r+$ $m)=r^{2}+m^{2}$. The trace works out to be

$$
\begin{aligned}
\operatorname{Tr}[]= & -\left(r^{2}+m^{2}\right)\left[\operatorname{Tr}\left\{\gamma^{5} \gamma^{\alpha} i \gamma r \gamma^{\mu} \gamma^{\nu}\right\}+\operatorname{Tr}\left\{\gamma^{5} \gamma^{\alpha} i \gamma p \gamma^{\mu} \gamma^{\nu}\right\}\right. \\
& \left.-\operatorname{Tr}\left\{\gamma^{5} \gamma^{\alpha} \gamma^{\mu} \gamma^{\nu} i \gamma q\right\}\right]-2 i r_{\nu} \operatorname{Tr}\left\{\gamma^{5} \gamma^{\alpha} i \gamma p \gamma^{\mu} i \gamma r\right\} \\
& +2 i r_{\mu} \operatorname{Tr}\left\{\gamma^{5} \gamma^{\alpha} i \gamma r \gamma^{\nu} i \gamma q\right\}+\operatorname{Tr}\left\{\gamma^{5} \gamma^{\alpha} i \gamma p \gamma^{\mu} i \gamma r \gamma^{\nu} i \gamma q\right\} \\
= & -4 i\left(r^{2}+m^{2}\right) \varepsilon_{\alpha \mu \nu \lambda}(r+p-q)_{\lambda}+8 i r_{\nu} \varepsilon_{\alpha \lambda \mu \kappa} p_{\lambda} r_{\kappa} \\
& +8 i r_{\mu} \varepsilon_{\alpha \lambda \nu \kappa} q_{\lambda} r_{\kappa}+\operatorname{Tr}\left\{\gamma^{5} \gamma^{\alpha} i \gamma p \gamma^{\mu} i \gamma r \gamma^{\nu} i \gamma q\right\} .
\end{aligned}
$$

Now we use the functions $B, C$ of ref. [7], but differing by a factor $i \pi^{2}$, for example

$$
B_{0}\left(p, m_{1}, m_{2}\right)=\int \mathrm{d}_{n} q \frac{1}{\left(q^{2}+m_{1}^{2}\right)\left((q-p)^{2}+m_{2}^{2}\right)}
$$

In terms of these functions, and suppressing mass dependence:

$$
\begin{aligned}
\frac{4 c_{\theta}}{i g^{3} s_{\theta}^{2}} \Gamma_{I}^{\alpha \mu \nu}= & -4 i \varepsilon_{\alpha \mu \nu \lambda}\left\{B_{1}(k) k_{\lambda}-B_{0}(k) q_{\lambda}\right\} \\
& +8 i \varepsilon_{\alpha \lambda \mu \kappa} p_{\lambda}\left\{C_{21} p_{\nu} p_{\kappa}+C_{22} k_{\nu} k_{\kappa}+C_{23}\left(p_{\nu} k_{\kappa}+k_{\nu} p_{\kappa}\right)+C_{24} \delta_{\nu \kappa}\right\} \\
& +8 i \varepsilon_{\alpha \lambda \nu \kappa} q_{\lambda}\left\{C_{21} p_{\mu} p_{\kappa}+C_{22} k_{\mu} k_{\kappa}+C_{23}\left(p_{\mu} k_{\kappa}+k_{\mu} p_{\kappa}\right)+C_{24} \delta_{\mu \kappa}\right\} \\
& +\operatorname{Tr}\left\{\gamma^{5} \gamma^{\alpha} i \gamma p \gamma^{\mu} i \gamma p \gamma^{\nu} i \gamma q\right\} C_{11}+\operatorname{Tr}\left\{\gamma^{5} \gamma^{\alpha} i \gamma p \gamma^{\mu} i \gamma k \gamma^{\nu} i \gamma q\right\} C_{12}
\end{aligned}
$$

All functions $C$ have the same functional dependence, i.e. $C=C(p, k, m, m, m)$. The remaining traces can now be done easily. For equal masses $B_{1}=-\frac{1}{2} B_{0}$, and one 
finds

$$
\begin{aligned}
\frac{4 c_{\theta}}{i g^{3} s_{\theta}^{2}} \Gamma_{I}^{\alpha \mu \nu}=i \varepsilon_{\alpha \mu \nu \lambda}\{ & -2 B_{0}(k)(p-q)_{\lambda}+8 C_{24}(p-q)_{\lambda} \\
& \left.+4 p^{2}\left(C_{11}-C_{12}\right) q_{\lambda}-4 q^{2} C_{12} p_{\lambda}\right\} \\
& +8 i \varepsilon_{\alpha \lambda \mu \kappa}\left\{\left(C_{22}+C_{12}\right) p_{\lambda} k_{\kappa} k_{\nu}+\left(C_{23}+C_{12}\right) p_{\lambda} k_{\kappa} p_{\nu}\right\} \\
& +8 i \varepsilon_{\alpha \lambda \nu \kappa}\left\{\left(C_{23}-C_{22}\right) p_{\lambda} k_{\kappa} k_{\mu}+\left(C_{21}-C_{23}+C_{11}-C_{12}\right) p_{\lambda} k_{\kappa} p_{\mu}\right\}
\end{aligned}
$$

To obtain the complete amplitude we must add on the same term, but with $p, \mu$ and $q, \nu$ interchanged. For the second diagram we get functions $\bar{C}$ defined by

$$
\bar{C}(p, q)=C(q, p)
$$

The following relations hold:

$$
\begin{gathered}
\bar{C}_{0}=C_{0} ; \quad \bar{C}_{11}=C_{11} ; \quad \bar{C}_{12}=C_{11}-C_{12} ; \\
\bar{C}_{22}=C_{21}+C_{22}-2 C_{23} ; \quad \bar{C}_{23}=C_{21}-C_{23} ; \quad \bar{C}_{24}=C_{24} .
\end{gathered}
$$

One so obtains

$$
\begin{aligned}
& \Gamma_{W}^{\alpha \mu \nu}=\Gamma_{I}^{\alpha \mu \nu}+\Gamma_{I I}^{\alpha \mu \nu} \\
&=\frac{-g^{3} s_{\theta}^{2}}{4 c_{\theta}}\left[\varepsilon _ { \alpha \mu \nu \lambda } \left\{-4 B_{0}(k)(p-k)_{\lambda}\right.\right. \\
&\left.\quad+16 C_{24}(p-q)_{\lambda}+8 p^{2}\left(C_{11}-C_{12}\right) q_{\lambda}-8 q^{2} C_{12} p_{\lambda}\right\} \\
& \\
& \quad+16 \varepsilon_{\alpha \lambda \mu \kappa} p_{\lambda} k_{\kappa}\left\{\left(C_{22}+C_{12}\right) k_{\nu}+\left(C_{23}+C_{12}\right) p_{\nu}\right\} \\
& \\
&\left.\quad+16 \varepsilon_{\alpha \lambda \nu \kappa} p_{\lambda} k_{\kappa}\left\{\left(C_{23}-C_{22}\right) k_{\mu}+\left(C_{21}-C_{23}+C_{11}-C_{12}\right) p_{\mu}\right\}\right]
\end{aligned}
$$

To verify the Ward identity we need to compute the diagrams of fig. 9. The corresponding equations are like those for the $\Gamma$ given before, but with $\gamma^{5} \gamma^{\alpha}$ replaced by $-2 c_{\theta}(m / M) \gamma^{5}$. The trace works out to $4 m \varepsilon_{\lambda \mu \nu \kappa} p_{\lambda} q_{\kappa}$. Adding the contribution of 


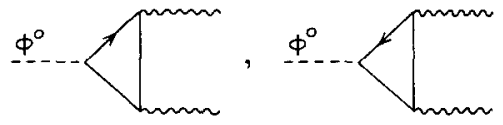

Fig. 9.

the second diagram of fig. 9 we find for the total of fig. 9 :

$$
\Gamma_{\phi}^{\mu \nu}=-\frac{4 i m^{2} g^{3} s_{\theta}^{2}}{M} \varepsilon_{\lambda \mu \nu \kappa} p_{\lambda} q_{\kappa} C_{0}
$$

The relevant $W$ ard identity derives from the gauge fixing term $-\partial_{\mu} W_{\mu}^{0}+M_{0} \phi^{0}$, with $M_{0}=M / c_{\theta}$. This implies

$$
i k_{\alpha} \Gamma_{W}^{\alpha \mu v}-\frac{M}{c_{\theta}} \Gamma_{\phi}^{\mu v}=0
$$

To evaluate this one uses the identity

$$
\begin{aligned}
C_{24} & =\frac{1}{4} i \pi^{2}-\frac{1}{2} m^{2} C_{0}+\frac{1}{4}\left\{B_{0}(k)-f_{1} C_{11}-f_{2} C_{12}\right\}, \\
f_{1} & =p^{2}, \quad f_{2}=p^{2}-(p+q)^{2}
\end{aligned}
$$

One obtains

$$
-\frac{i g^{3} s_{\theta}^{2}}{4 c_{\theta}} \varepsilon_{\alpha \mu \nu \lambda} k_{\alpha} p_{\kappa}\left\{8 i \pi^{2}-16 m^{2} C_{0}\right\}+\frac{4 i m^{2} g^{3} s_{\theta}^{2}}{c_{\theta}} \varepsilon_{\lambda \mu \nu \kappa} p_{\lambda} q_{\kappa} C_{0}=\frac{2 \pi^{2} g^{3} s_{\theta}^{2}}{c_{\theta}} \varepsilon_{\alpha \mu \nu \lambda} k_{\alpha} p_{\lambda} .
$$

This is non-zero, which is the anomaly. The anomaly would be removed if $\Gamma_{\phi}^{\mu \nu}$ had the additional term:

$$
-\frac{2 g^{3} s_{\theta}^{2} \pi^{2}}{M} \varepsilon_{\lambda \mu \nu \kappa} p_{\lambda} q_{\kappa}
$$

which corresponds to a term

$$
\frac{i g^{3} s_{\theta}^{2}}{16 \pi^{2} M} \varepsilon_{\lambda \mu \kappa \nu} \partial_{\lambda} A_{\mu} \partial_{\kappa} A_{\nu} \phi^{0}
$$

in the lagrangian (multiply by $(2 \pi)^{4} i$, and remember that each derivative gives a factor $i$ ). Note that this term is real, because one of the indices of the $\varepsilon$ tensor is 4 and $\partial_{4}$ and $A_{4}$ are imaginary with our convention for the metric.

This is only one of the anomalous graphs in the standard weak model. Using the formula for all anomalies given by Bardeen [8] we deduce the term that will remove 
all anomalies in one loop:

$$
\frac{i g^{3} \tan \theta}{128 \pi^{2} M} \varepsilon_{\lambda \mu \kappa \nu}\left(F_{\lambda \mu} G_{\kappa \nu}^{a} \phi^{a}+F_{\lambda \mu} F_{\kappa \nu} \phi^{0} \tan \theta\right)
$$

where $F_{\lambda \mu}$ is the $\mathrm{U}(1)$ field strength, $\partial_{\lambda} B_{\mu}^{0}-\partial_{\mu} B_{\lambda}^{0}, G_{\kappa \nu}^{a}$ is the $\mathrm{SU}(2)$ field strength, $\partial_{\kappa} B_{\nu}^{a}-\partial_{\nu} B_{\kappa}^{a}+g \varepsilon^{a b c} B_{\kappa}^{b} B_{\nu}^{c}$, and $\phi^{a}$ is the unphysical Higgs. We can expand this to find all the effective vertices necessary to restore the Ward identities; removing a factor $\left(g^{3} \pi^{2} / M\right) \varepsilon_{\lambda \mu \kappa \nu}$ from each vertex we have:

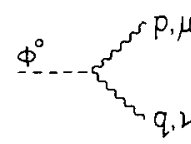

$$
2 s_{\theta}^{2} p_{\lambda} q_{k}
$$
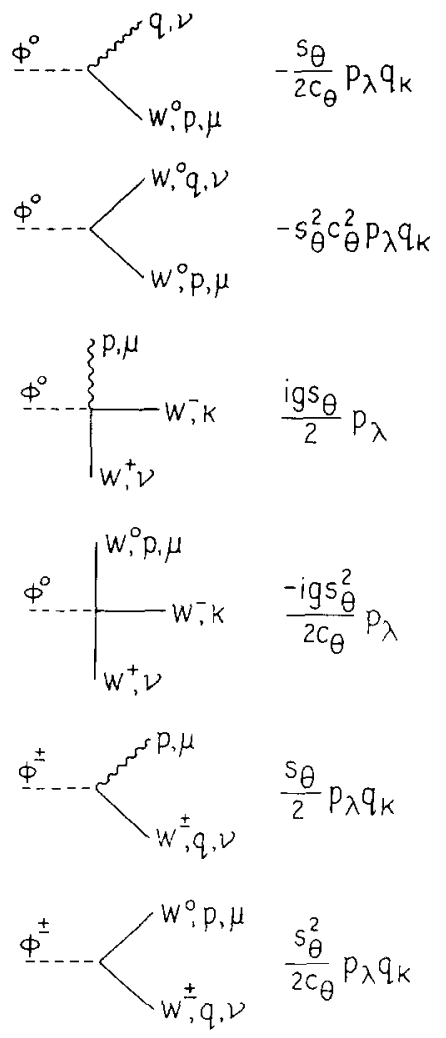

$$
\Phi^{ \pm} \sum_{W^{ \pm}, k}^{p, \mu} \quad \pm \frac{i g s_{\theta}^{2}}{2}\left(p_{\lambda}-q_{\lambda}\right)
$$




$$
\begin{aligned}
& \Phi^{ \pm}\left\{\begin{array}{l}
q, v \\
W^{ \pm}, k
\end{array} w^{0}, p, \mu \quad \mp \frac{i g s_{\theta}}{2 c_{\theta}}\left(s_{\theta}^{2} p_{\lambda}+c_{\theta}^{2} q_{\lambda}\right)\right. \\
& \left.\Phi^{ \pm}\right|_{W^{ \pm}, K} ^{W^{0} p, \mu} w_{, q, \nu}^{\circ} \quad \mp \frac{i g s_{\theta}^{2}}{2}\left(p_{\lambda}-q_{\lambda}\right)
\end{aligned}
$$

\section{Appendix B}

FEYNMAN RULES FOR "SUPERSYMMETRIC" FERMION AND BOSON INTERACTION WITH THE HIGGS BOSON

Following the conventions in Passarino and Veltman [7] we can add the Feynman rules for $\mathrm{u}$ and $\mathrm{d}$ quark interactions with the Higgs. Using the lagrangian, eq. (4.1), and letting

$$
\begin{gathered}
\psi_{\mathrm{L}}=\left(\begin{array}{l}
d_{\mathrm{L}} \\
u_{\mathrm{L}}
\end{array}\right), \quad \psi_{\mathrm{R} 1}=u_{\mathrm{R}}, \quad \psi_{\mathrm{R} 2}=d_{\mathrm{R}}, \\
H=\left(\begin{array}{c}
i \phi^{-} \\
\frac{Z+i \phi^{3}+V}{\sqrt{2}}
\end{array}\right), \quad A=\left(\begin{array}{l}
A_{1} \\
A_{2}
\end{array}\right),
\end{gathered}
$$

and eliminating $F$ and $f_{i}$, we can then find the Feynman rules for these fields:

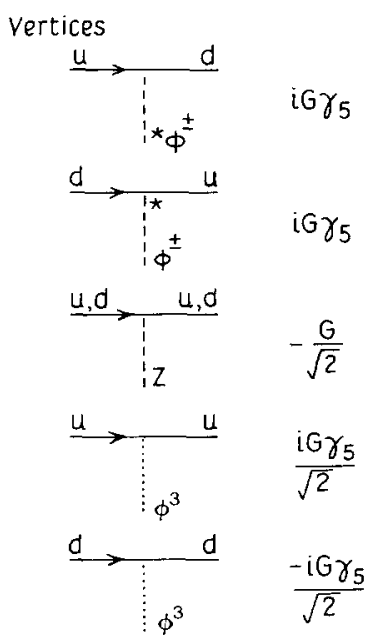



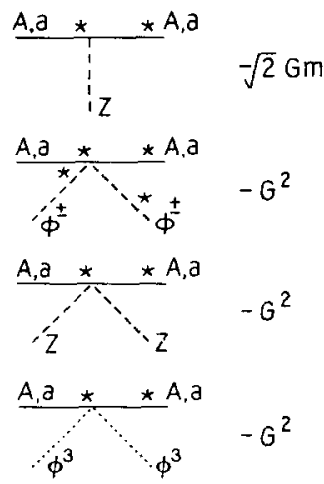

Propagators

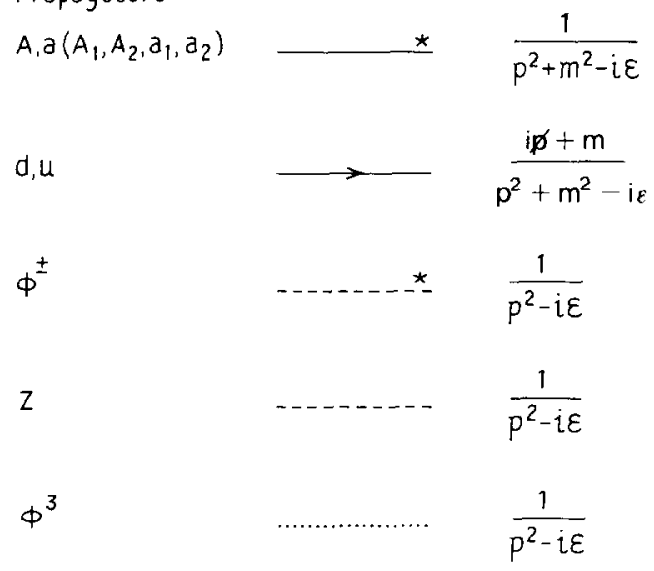

\section{Appendix C}

RESIDUAL EFFECTS OF HEAVY FERMIONS

The simplest graph to calculate is the Higgs self-energy graph [11], using the rules of appendix B and ref. [7]:

$$
\begin{aligned}
& \Gamma_{2}^{\mathrm{f}}=-\int \mathrm{d}_{n} p \frac{\operatorname{Tr}[(-i(q+p)+m(-i p+m)]}{\left((q+p)^{2}+m^{2}\right)\left(p^{2}+m^{2}\right)}\left(\frac{G^{2}}{2}\right) \\
& =-\int_{0}^{1} \mathrm{~d} x \frac{2 G^{2} i \pi^{n / 2} \Gamma\left(2-\frac{1}{2} n\right)}{\left(m^{2}+x(1-x) q^{2}\right)^{1-n / 2}}\left(\frac{1-n}{1-\frac{1}{2} n}\right) .
\end{aligned}
$$


We can expand this in a Laurent series in $\varepsilon=4-n$ and $m$ to find the behavior as $m$ gets large:

$$
\begin{aligned}
\Gamma_{2}^{\mathrm{f}}= & G^{2}\left[-6 m^{2}\left[\frac{2 \pi^{2}}{\varepsilon}+C-\pi^{2} \ln m^{2}+\frac{1}{3} \pi^{2}\right]\right. \\
& \left.-q^{2}\left[\frac{2 \pi^{2}}{\varepsilon}+C-\pi^{2} \ln m^{2}-\frac{2}{3} \pi^{2}\right]+\frac{\pi^{2}}{10} \frac{q^{4}}{m^{2}}+\cdots\right],
\end{aligned}
$$

where $C$ is a constant. Since $G=m / V$ the term quartic in $q$ is constant as $m$ gets large.

This result can be used to calculate the corrections to the three-Higgs interaction using the fact that

$$
\frac{G}{\sqrt{2}}\left(\frac{1}{i p+m}\right)^{2}=-\frac{G}{\sqrt{2}} \frac{\partial}{\partial m}\left(\frac{1}{i p+m}\right)
$$

to calculate the graphs

$$
\Gamma_{30}^{\mathrm{f}}=-\frac{G}{\sqrt{2}} \frac{\partial}{\partial m} \Gamma_{2}^{\mathrm{f}}=\frac{G^{3}}{\sqrt{2}}\left[12 m\left[\frac{2 \pi^{2}}{\varepsilon}-\pi^{2} \ln M^{2}-\frac{2 \pi^{2}}{3}\right]-2 \pi^{2} \frac{q^{2}}{m}+\cdots\right] .
$$

Then we can use Bose symmetry to deduce that the graphs

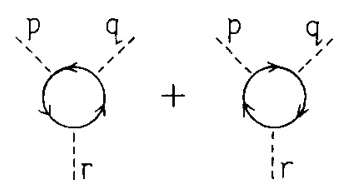

are : $\quad \Gamma_{3}^{\mathrm{f}}=\frac{G^{3}}{\sqrt{2}}\left[12 m\left[\frac{2 \pi^{2}}{\varepsilon}-\pi^{2} \ln m^{2}-\frac{2 \pi^{2}}{3}\right]-\pi^{2} \frac{\left(p^{2}+q^{2}+r^{2}\right)}{m}\right]$.

This is the lowest order correction which increases with $m$ and cannot be absorbed into the renormalization. 
The scalar partners have two one-loop corrections to the propagator:

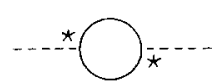

(a)

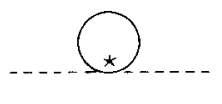

(b)

Expanding as before:

$$
\begin{aligned}
\Gamma_{2 \mathrm{a}}^{\mathrm{s}} & =2 G^{2} m^{2} \int \mathrm{d}_{n} p \frac{1}{\left(p^{2}+m^{2}\right)\left((p+q)^{2}+m^{2}\right)} \\
& =2 G^{2} m^{2} \int \mathrm{d} x \frac{i \pi^{n / 2} \Gamma\left(2-\frac{1}{2} n\right)}{\left(m^{2}+x(1-x) q^{2}\right)^{2-n / 2}}, \\
\Gamma_{2 \mathrm{~b}}^{\mathrm{s}} & =-G^{2} \int \mathrm{d}_{n} p \frac{1}{\left(p^{2}+m^{2}\right)}=\frac{-G^{2} i \pi^{n / 2} \Gamma\left(2-\frac{1}{2} n\right)}{\left(1-\frac{1}{2} n\right)\left(m^{2}\right)^{1-n / 2}}, \\
\Gamma_{2}^{\mathrm{s}} & =G^{2}\left[3 m^{2}\left[\frac{2 \pi^{2}}{\varepsilon}+C-\pi^{2} \ln m^{2}+\frac{1}{3} \pi^{2}\right]-\frac{1}{3} \pi^{2} q^{2}+\frac{\pi^{2}}{30} \frac{q^{4}}{m^{2}}+\cdots\right] .
\end{aligned}
$$

Notice that 2 of these charged scalars will cancel the mass renormalization necessary for 1 fermion, but not the higher terms in $q^{2}$.

The three-Higgs graph can be found as before:

$$
\Gamma_{3}^{\mathrm{s}}=\frac{1}{2} G^{2}\left[-6 m\left[\frac{2 \pi^{3}}{\varepsilon}+C-\pi^{2} \ln m^{2}+\frac{1}{3} \pi^{2}\right]+\cdots\right] .
$$

For the scalar there is no $\left(p^{2}+q^{2}+r^{2}\right)$ term.

\section{References}

[1] T. Appelquist and J. Carazzone, Phys. Rev. D11 (1975) 2856

[2] Y. Kazama, D. Unger and Y.-P. Yao, Univ. of Michigan preprint UM HE 80-36

[3] S. Weinberg, Phys. Rev. Lett. 19 (1967) 1264

[4] S. Raby, S. Dimopoulos and L. Susskind, Nucl. Phys. B169 (1980) 373

[5] G. 't Hooft, Utrecht PRINT-80-0083, Lecture Cargese Summer Inst.

[6] G. 't Hooft and M. Veltman, Nucl. Phys. B44 (1972) 189

[7] G. Passarino and M. Veltman, Nucl. Phys. B160 (1979) 151

[8] W. Bardeen, Phys. Rev. 184 (1969) 1848

[9] S. Bludman, Nuovo Cim. 9 (1958) 433;

S. Glashow, Nucl. Phys. 22 (1961) 579;

S. Weinberg, Remark at 1967 Solvay Conf., Talk of H. Durr; Phys. Rev. Lett. 19 (1967) 1264;

S. Glashow, J. Illiopoulos and L. Maiani, Phys. Rev. D2 (1970) 1285;

C. Bouchiat, J. Iliopoulos and Ph. Meyer, Phys. Lett. 42B (1972) 91 
[10] P.Q. Hung, Phys. Rev. Lett. 42 (1979) 873;

S. Wolfram and H.D. Politzer, Phys. Lett. 82B (1979) 242

[11] M.S. Chanowitz, M.A. Furman and I. Hinchliffe, Nucl. Phys. B153 (1979) 402

[12] S.Y. Lee and A.M. Sciaccaluga, Nucl. Phys. B96 (1975) 435

[13] F. Wilczek, Phys. Rev. Lett. 39 (1977) 1304 